LAWRENCE LIVERMORE N A T IO N A L LABORATORY

Numerical Investigation of

Transients in the SSPX Spheromak

C.R. Sovinec, B.I. Cohen, G.A. Cone, E.B. Hooper, H.S. McLean

October 18, 2004

Physical Review Letters 
This document was prepared as an account of work sponsored by an agency of the United States Government. Neither the United States Government nor the University of California nor any of their employees, makes any warranty, express or implied, or assumes any legal liability or responsibility for the accuracy, completeness, or usefulness of any information, apparatus, product, or process disclosed, or represents that its use would not infringe privately owned rights. Reference herein to any specific commercial product, process, or service by trade name, trademark, manufacturer, or otherwise, does not necessarily constitute or imply its endorsement, recommendation, or favoring by the United States Government or the University of California. The views and opinions of authors expressed herein do not necessarily state or reflect those of the United States Government or the University of California, and shall not be used for advertising or product endorsement purposes. 


\title{
Numerical Investigation of Transients in the SSPX Spheromak
}

\author{
C. R. Sovinec, ${ }^{1}$ B. I. Cohen, ${ }^{2}$ G. A. Cone, ${ }^{1}$ E. B. Hooper, ${ }^{2}$ and H. S. McLean ${ }^{2}$ \\ ${ }^{1}$ University of Wisconsin, Madison, Wisconsin 53706 \\ ${ }^{2}$ Lawrence Livermore National Laboratory, Livermore, California 94550
}

\begin{abstract}
Nonlinear simulations of plasma discharges in the Sustained Spheromak Physics Experiment demonstrate the role of transient effects in establishing a toroidal magnetic structure that confines internal energy. The magnetohydrodynamics-based model includes collisional anisotropic thermal energy transport and temperature-dependent electrical resistivity that are realistic for the open-field regions of the plasma. The modeling shows that while dynamo activity is responsible for generating net poloidal flux during the formation current pulse, it is insufficient to sustain the configuration during the quiescent phase. The second current pulse improves confinement by keeping the $q$ profile from falling significantly below the value of $1 / 2$, thereby suppressing resonant $\mathrm{m}=1, \mathrm{n}=2$ magnetohydrodynamic activity. Direct comparisons of laboratory observations and simulation results validate essential aspects of the model.
\end{abstract}

PACS numbers: 52.55.Ip, 52.65.Kj, 52.35.Py

Magnetic reconnection and relaxation occur to some degree in nearly all magneticconfinement configurations, but they are elemental processes for electrostatically driven spheromaks. The plasma conducts electrical current between electrodes that are linked by open magnetic field-lines, and nonlinear effects relax the current to a desirable profile through reconnection and the conversion of toroidal magnetic flux into poloidal flux $[1,2,3]$. While 
inductive effects can contribute to formation, sustaining spheromak plasmas by DC injection requires dynamo activity from symmetry-breaking magnetic fluctuations [4,5]. Analytical work on the magnetohydrodynamic (MHD) processes underlying electrostatic formation and sustainment led to relaxation and statistical theories [6,7], which provide physical insight regarding the importance of nonlinear effects. Experimentally, observations of electric fields associated with MHD dynamo in the SPHEX device indicate power redistribution from the open current column to the amplified-flux region [8]. Numerically, nonlinear three-dimensional (3D) resistive MHD computations without temperature evolution reproduce the flux conversion process and electrostatic sustainment from first-principles [9]. However, until now, a consistent theoretical calculation of the magnetic evolution and energy confinement has not been performed. The earlier computations predicted chaotic scattering of open magnetic field lines throughout the plasma in typical driven conditions [10]. In such open-field configurations, electron temperatures resulting from Ohmic heating with classical parallel transport are limited to tens of electron-Volts [11,12], whereas much higher temperatures have been observed. In some cases, like the record $400 \mathrm{eV}$ observation [13], the highest temperature clearly occurs subsequent to the electrostatic injection pulse, and the earlier computations have shown that decay can effect a topological change to closed magnetic-flux surfaces while inductive Ohmic heating continues [9]. Temperatures exceeding $100 \mathrm{eV}$ have also been reported in the Sustained Spheromak Physics Experiment (SSPX) [14] upon application of a second current drive pulse that follows a brief period of decay $[15,16]$.

Whether transient effects are important for energy confinement in SSPX is the subject of our recent numerical investigation. We consider a single-fluid model in a domain that is based on the SSPX vacuum chamber and apply a simulated injector-current waveform that approximates 
the DC drive used in discharges numbered 4620-4662 [15]. The temperature computation includes anisotropic, temperature-dependent thermal conduction, and the magnetic-field computation includes temperature-dependent electrical resistivity. As a consequence, the temperature and magnetic fields are tightly coupled; magnetic topology and parallel thermal conduction regulate energy confinement, while resistivity influences magnetic reconnection and diffusion. In addition, the length of open magnetic field lines and the extent of any region of closed magnetic flux are governed dynamically by MHD instabilities, which respond quickly to changes in the parallel current density distribution.

Our simulations of SSPX solve nonlinear time-dependent equations for particle number density $\left(n_{i}=n_{e}=n\right.$ with quasineutrality), plasma flow velocity $(\mathbf{V})$, temperature (assuming $T_{i}=T_{e}=T$ ), and magnetic field (B). In MKS units, the evolution equations are

$$
\begin{aligned}
& \frac{\partial n}{\partial t}+\nabla \cdot(n \mathbf{V})=\nabla \cdot D \nabla n \\
& \rho\left(\frac{\partial \mathbf{V}}{\partial t}+\mathbf{V} \cdot \nabla \mathbf{V}\right)=\mathbf{J} \times \mathbf{B}-\nabla p+\nabla \cdot \rho v \nabla \mathbf{V} \\
& \frac{n k}{\gamma-1}\left(\frac{\partial T}{\partial t}+\mathbf{V} \cdot \nabla T\right)=-\frac{p}{2} \nabla \cdot \mathbf{V}+\nabla \cdot n k\left[\chi_{\|} \hat{\mathbf{b}} \hat{\mathbf{b}}+\chi_{\perp}(\mathbf{I}-\hat{\mathbf{b}} \hat{\mathbf{b}})\right] \nabla T+\frac{\eta \mathbf{J}^{2}}{2} \\
& \frac{\partial \mathbf{B}}{\partial t}=\nabla \times(\mathbf{V} \times \mathbf{B}-\eta \mathbf{J}) \\
& \mu_{0} \mathbf{J}=\nabla \times \mathbf{B}
\end{aligned}
$$

where $p \equiv 2 n k T$ is the sum of electron and ion pressures, and $\hat{\mathbf{b}} \equiv \mathbf{B} /|\mathbf{B}|$ is the evolving magnetic direction vector field. The simulations consider $n, T, \mathbf{V}, \mathbf{B}$, and $\mathbf{J}$ to be functions of all three 
spatial dimensions and time, so magnetic fluctuations and anisotropic heat flow are modeled explicitly. The parallel and perpendicular thermal diffusivities are $\chi_{\|}=387 T^{5 / 2} \mathrm{~m}^{2} / \mathrm{s}$ and $\chi_{\perp}=0.50 T^{-1 / 2} B^{-2} \mathrm{~m}^{2} / \mathrm{s}$ ( $B$ in Tesla) based on electrons and ions [17], respectively, for a hydrogen plasma at $n=5 \times 10^{19} \mathrm{~m}^{-3}$. The numerical computation of $\chi_{\perp}$ is simplified by using the toroidal average (indicated by \langle\rangle , hereafter) of the evolving temperature and magnetic induction fields. Similarly, the electrical diffusivity is computed as $\eta / \mu_{0}=411(1 \mathrm{eV} /\langle T\rangle)^{3 / 2} \mathrm{~m}^{2} / \mathrm{s}$. (Numerical tests show no significant deviation from results with a 3D computation of resistivity in the conditions of interest.) An isotropic viscosity ( $v)$ of $2000 \mathrm{~m}^{2} / \mathrm{s}$ is used to provide nonlinear numerical stability during the full-power stage of the evolution. With temperatures of approximately $30 \mathrm{eV}$ during this stage, the Lundquist number (computed as $\mathrm{S}=\mu_{0} R v_{A} / \eta$, where $R$ is the radius of SSPX and $v_{A}$ is the Alfvén speed) is of order $10^{6}$. This is much larger than values considered in the earlier simulations of sustainment [9], and the relatively large magnetic Prandtl number $\left(\mathrm{Pm} \equiv \mu_{0} v / \eta\right)$ maintains a numerically tractable Hartmann number $\left(\mathrm{H} \equiv \mathrm{Pm}^{-1 / 2} \mathrm{~S}\right)$ of order $10^{4}$. The diffusion term in Eq. (1) is a substitute for particle transport and atomic fueling effects that are not present in the MHD model. The artificial diffusivity $(D)$ is also set to $2000 \mathrm{~m}^{2} / \mathrm{s}$ to help keep the computed minimum of the number density field above zero during the violent full-power stage. For the same reason, the diffusivity is increased locally in computational cells where $n$ falls to $3 \%$ of its volume-average value.

The conductive heat-flux model applied in our MHD simulations is appropriate for collisional plasmas [17] with rapid electron-ion thermal equilibration; parallel (perpendicular) conduction is computed from the relation for electrons (ions). Near the electrodes, the temperature is on the order of an electron-Volt or less, so the edge plasma is very collisional. In 
the interior, magnetic field that links the electrodes allows rapid parallel conduction to the edge and tends to keep the plasma collisional. Since the effective collisional mean-free-path is $4 \times 10^{-4} T^{2} \mathrm{~m}\left(T\right.$ in eV) [17] for the experimentally measured particle number density $n \cong 5 \times 10^{19}$ and singly charged ions, its value is less than the radius of $\operatorname{SSPX}(R=0.5 \mathrm{~m})$ for $T<35 \mathrm{eV}$. Larger temperatures, and hence less collisional conditions, only occur where the open field-lines are very long [11] or where closed flux-surfaces form. Since our primary interest is the dynamics that lead to improving energy confinement, the collisional behavior is most important. Where high-temperature conditions are successfully achieved, neglecting kinetic effects will tend to over-predict parallel heat flux $[18,19]$, and neglecting neoclassical and turbulence effects will underestimate ion perpendicular heat transport in closed-field regions. However, thermal equilibration among electrons and ions becomes slow with respect to the MHD dynamics, and electron perpendicular heat flux may be over-estimated by the single-temperature model.

The system of nonlinear equations (1-5) is solved numerically as an initial-value problem with the NIMROD code, which uses a high-order spatial representation to resolve anisotropies [20]. Our computational domain in these simulations models the SSPX flux conserver and the downstream end of its plasma gun with 1152 bicubic finite elements. For the toroidal direction, our finite Fourier series representation includes only the $n=0$ component when modeling the symmetric ejection of plasma from the gun, the $0 \leq n \leq 2$ components for the brief formation period, and $0 \leq n \leq 5$ components for the decay and second current pulse. The initial conditions include a distribution of poloidal magnetic flux (see Fig. 1a) that is similar to the bias-flux generated by coils located outside the SSPX vacuum chamber; this open flux remains tied to the electrodes for the time-scale of SSPX discharges. The computational domain does not represent the entire plasma gun region of SSPX, so the boundary at the upstream end is artificial. For 
convenience, we model it as a solid wall, noting that the MHD dynamics of interest occur away from this boundary. The tangential component of electric field, $\mathbf{E} \times \hat{\mathbf{n}}$, is set to zero on the electrode surfaces, and a time-dependent boundary condition on the toroidal component of $\langle\mathbf{B}\rangle$ along the artificial boundary is used to inject power and magnetic helicity. In SSPX, the injected current is controlled over the entire discharge, and the potential difference between the electrodes is measured. Injector current is also specified in the computations, except during the currentdecay phase between the two applied pulses, which is simulated as a temporary short-circuit. A final point related to the injector current is that while radiation amounts to a small fraction of the power loss in SSPX [15] and is therefore not modeled, a sink of internal energy is imposed in computational cells that are adjacent to the artificial upstream boundary. Here, the simulated plasma remains at the imposed wall temperature of $0.1 \mathrm{eV}$ to control the thickness of the resistive boundary layer and allow the discharge to lift off of the artificial surface when power is applied. Very little poloidal flux traverses this layer, so parallel thermal conduction tends to deposit energy on other external surfaces.

As shown in Fig. 2a, the injector-current waveform is modeled as increasing linearly over the first $0.1 \mathrm{~ms}$, constant at $400 \mathrm{kA}$ for the next $0.02 \mathrm{~ms}$, decaying until $0.5 \mathrm{~ms}$, and then constant at $200 \mathrm{kA}$. The resulting parallel current density profile is initially stable to toroidally asymmetric perturbations as mass and poloidal flux are swept out of the gun. While the profile is still stable but becoming pinched at $t=0.08 \mathrm{~ms}$, the $\mathrm{n}=1$ and $\mathrm{n}=2$ Fourier components are introduced with velocity perturbations amounting to $2 \mathrm{~J}$ of kinetic energy. At $t \cong 0.09 \mathrm{~ms}$, the current profile becomes unstable to an $\mathrm{n}=1 \mathrm{MHD}$ mode, and magnetic fluctuations subsequently grow at a rate of $5 \times 10^{5} \mathrm{~s}^{-1}$, which is fast relative to the injector-current transient. Abrupt changes in toroidal plasma current and magnetic energy, evident at $t=0.1 \mathrm{~ms}$ in Figs. $2 \mathrm{c}-\mathrm{d}$, occur when the $\mathrm{n}=1$ mode 
saturates and toroidal flux is converted into poloidal flux $[1,9,21]$. The flux conversion extends the range of poloidal flux by $200 \%$ during the evolution from the unstable pinch state (Fig. 1b) to the saturated state (Fig. 1c), but the injector voltage is very large $(>1 \mathrm{kV})$ in both the simulation and the experiment (see Fig. 2b). In contrast, the voltage during the second current-drive pulse is much smaller, $20 \mathrm{~V}$ in the simulation and fluctuating around $200 \mathrm{~V}$ in the experiment, where the latter includes the sheath potential, which amounts to approximately $100-150 \mathrm{~V}[11,22]$. The power injected with the second current pulse is, therefore, only a small fraction of the power used during formation. Nonetheless, the rates of decay of toroidal current and magnetic energy are reduced (Figs 2c-d) relative to the period between the two injector-current pulses. Although it is not possible to measure toroidal current and magnetic energy in SSPX directly, we are able to fit MHD equilibria to various laboratory measurements after $t=0.3 \mathrm{~ms}$ using the CORSICA code [14]. A comparison of results from the 3D simulations and from a sequence of fitted equilibria is shown in Figs. 2c-d. Over the second current-drive pulse, there is agreement to within $25 \%$ for the toroidal current, the magnetic energy, and their rates of decay.

The magnetic fluctuations are largest during the $400 \mathrm{kA}$ formation pulse, when the $\mathrm{n}=1$ mode is relaxing the configuration, and decay rapidly during the subsequent ramp-down. They evolve to their lowest levels during the second current pulse, as shown in Fig. 3. In fact, the relative fluctuation in the poloidal component of $\mathbf{B}$ measured on the outer wall at the midplane $(Z=0)$ drops below $1 \%$ in both the experiment and the simulation (Fig. 3a). The $\mathrm{n}=2$ mode is particularly responsive to the second current pulse. Figure $3 \mathrm{~b}$ shows traces of volume-integrated magnetic fluctuation energy from the simulation, together with results from another simulation where the second current pulse is not applied. The energy in the $n=2$ component decays until $t>0.8 \mathrm{~ms}$ in the simulation with the second current pulse. When current is just allowed to decay, 
the $\mathrm{n}=2$ and $\mathrm{n}=4$ Fourier components grow to much larger levels from $t=0.6 \mathrm{~ms}$ onward. The 'quiescent' period brought about by the second current pulse correlates with increasing temperatures, as reported for the experiment in Ref. [15]. Figure 4a shows a comparison of Thomson-scattering measurements of central $T_{e}$ recorded over the laboratory discharge series with the evolution of maximum $\langle T\rangle$ from the simulations. While the experiment produced a sharper positive response at $t=1 \mathrm{~ms}$, reaching a maximum of $120 \mathrm{eV}$ vs. $75 \mathrm{eV}$ in the simulation, it is clear that temperatures also rise substantially with the decline of magnetic fluctuations in the simulation. Furthermore, without the second current pulse, the single-fluid model produces a maximum temperature of only $49 \mathrm{eV}$. Temperature profiles (Fig. 4b) show that energy confinement occurs in a toroidal region, which surrounds relatively cold plasma along the geometric axis.

The discharge essentially appears to be in a sustained state during application of the second injector pulse (see Figs. 2c-d); however, indefinite sustainment of $\left\langle J_{\phi}\right\rangle$ against resistive dissipation in the region of amplified poloidal flux requires dynamo activity [4]. The existence of dynamo activity in simulations of sustained conditions has been demonstrated with the simpler " $0-\beta$ " MHD model [9], but it is accompanied by large $\mathrm{n}=1$ fluctuations and chaotic scattering of magnetic fields. The present set of simulations exhibits similar behavior only during the first injector-current pulse, as shown by the MHD dynamo power density in Fig. 5. Here, we are considering $-\langle\mathbf{v} \times \mathbf{b}\rangle \cdot\langle\mathbf{J}\rangle$ as a fluctuation-induced contribution to $\langle\mathbf{E}\rangle \cdot\langle\mathbf{J}\rangle$ in the evolution of energy density associated with the symmetric component of $\mathbf{B}$,

$$
\frac{1}{2 \mu_{0}} \frac{\partial\langle B\rangle^{2}}{\partial t}+\nabla \cdot\langle\mathbf{E}\rangle \times\langle\mathbf{B}\rangle=-\langle\mathbf{E}\rangle \cdot\langle\mathbf{J}\rangle
$$


from Fararday's law. (Lower-case vector notation indicates the sum of $\mathrm{n}>0$ Fourier components.) Near the axis of the amplified poloidal flux, $R \cong 0.35 \mathrm{~m}$, the magnitude of the dynamo power density during the first pulse $(t=0.12 \mathrm{~ms})$ reaches $10^{9} \mathrm{~W} / \mathrm{m}^{3}$, whereas with $\langle J\rangle \cong 2.5 \mathrm{MA} / \mathrm{m}^{2}$ and $\eta=3.1 \times 10^{-6} \mathrm{Ohm}-\mathrm{m}$ at $\langle T\rangle=30 \mathrm{eV}$, the resistive power density is only $2 \times 10^{7} \mathrm{~W} / \mathrm{m}^{3}$. The toroidal component of $-\langle\mathbf{v} \times \mathbf{b}\rangle$ also exceeds $\eta\left\langle J_{\phi}\right\rangle$ by a factor of 50, so the amount of amplified poloidal flux is still increasing at this time. During the second injectorcurrent pulse $(t=1.2 \mathrm{~ms})$, the dynamo power density is more than two orders of magnitude smaller, and in the vicinity of the magnetic axis, it is less than $5 \times 10^{5} \mathrm{~W} / \mathrm{m}^{3}$. The current density at this location is essentially unchanged, and even considering the large increase in temperature, the resistive power density is $3 \times 10^{6} \mathrm{~W} / \mathrm{m}^{3}$. Thus, any possible dynamo activity is far less than what is required to sustain the current; though, the instantaneous resistive decay rate is slow relative to the injector transients.

Through anisotropic thermal energy transport, the temperature evolution provides an indirect but sensitive gauge of the magnetic topology. In Figure 6, we compare temperature profiles and Poincaré plots of the magnetic field at the same toroidal angle $(\phi)$ during the first and second current pulses. During the first pulse (Figs. 6a-b), the magnetic topology exhibits chaotic scattering, as found in the earlier simulation study of spheromak sustainment [10] and in related computations for DC current injection with a toroidal bias field [23]. The sparsity of punctures in Fig. 6a indicates that the traced field-lines complete only a small number of toroidal transits before encountering an electrode surface. The computed maximum temperature of $35 \mathrm{eV}$ is consistent with an analytical prediction for temperature on open magnetic field-lines subject to Ohmic heating and parallel thermal conduction at a fixed current density [11]. For the plasma 
parameters listed previously, a current density magnitude of $8 \mathrm{MA} / \mathrm{m}^{2}$ (taken from the geometric axis), and a parallel connection-length estimate of $3 \mathrm{~m}$, the analytical relation Eq. (19) from Ref. [11] predicts a maximum temperature of $30 \mathrm{eV}$.

When the primary drive is removed, the magnetic topology changes quickly. Without the large source of power, the edge and geometrically central regions cool rapidly via parallel conduction, making them very resistive. This enhances magnetic reconnection and diffusion in these regions, and it helps remove the current-gradient drive of the $n=1$ mode. The length of field-lines that pass through the toroidal region of amplified poloidal flux then increases, leading to the start of increasing temperatures shown in Fig. 4a. Application of the second current pulse at $t=0.5 \mathrm{~ms}$ and the resulting quiescent period produce large closed-flux surfaces at $t=1.2 \mathrm{~ms}$, as evident in Fig. 6c. Ohmic heating continues, due to gradual resistive decay, but energy loss involves slower cross-field transport. This results in a large temperature gradient at the edge of the magnetically closed region (Fig. 6d) and the largest temperature over the entire simulation (Fig. 4). The late-time Poincaré plot also shows an $m=2$ island structure from the $n=4$ magneticfield component that lies near the edge of the closed-flux region and an $\mathrm{m}=2$ structure from the $\mathrm{n}=3$ component near the magnetic axis. Between the two island chains is a region of stochastic magnetic field. The influence of the two island structures and the stochastic region are evident in the constant-temperature contours shown in Fig. $6 \mathrm{~d}$.

It is clear that the magnetic fluctuations with Fourier components $n>1$ have a strong influence on the evolution of temperature and that the second current pulse provides a means of transiently controlling the $n=2$ component. The beneficial effect of the second current pulse can be understood from the safety factor $(q)$ profiles shown in Fig. 7. The $q$-computation is based on the toroidally symmetric fields, $q=d\langle\Phi\rangle / d\langle\Psi\rangle$, where $\langle\Phi\rangle$ and $\langle\Psi\rangle$ are toroidal and poloidal 
magnetic flux functions of $\langle\mathbf{B}\rangle$, respectively. Although the profile cannot be interpreted as a magnetic winding number at $t=0.12 \mathrm{~ms}$ when the field is open and chaotic, it does indicate that the toroidal flux is greatest during the initial pulse. When the injector current and magnetic fluctuations decrease (and the sense of a magnetic winding number becomes more appropriate), the $q$-values fall, particularly near the edge of the amplified flux. Without the second current pulse, the outer $q$-values are well below 0.5 , and the $\mathrm{m}=1, \mathrm{n}=2$ mode is resonant near the middle of the amplified flux region. In contrast, application of the second current pulse retains more toroidal flux, so that the $\mathrm{m}=1, \mathrm{n}=2$ mode is not resonant until later in time and then only near the edge of the amplified flux region. The tendency of excluding poloidal flux from the gun (if not the existence of a separatrix) when current is injected makes the $q$-profile rise sharply at the edge of the amplified flux region. By noting the position of the closed-flux region in Fig. $6 \mathrm{c}$ and comparing it to the extent of the amplified-flux region in Fig. 1d, it is evident that a nontrivial fraction of the amplified flux remains open. Thus, the $m=2, n=4$ island structure lies along the inner of the two $q=0.5$ surfaces, so the large closed-flux surfaces, which form a transport barrier, are at or near the minimum of the $q$-profile. Near the magnetic axis, our $q$-value computation loses accuracy, but the fact that its value is near 0.67 is consistent with the existence of the $m=2$, $\mathrm{n}=3$ island structure in Fig. $6 \mathrm{c}$.

In assessing the MHD/collisional heat-flux modeling of these SSPX discharges, we note that quantitative results on driven plasma current, stored magnetic energy, local magnetic fluctuation levels, and temperatures compare fairly well over most of the discharge. The largest discrepancy is in the evolution of temperature when it climbs rapidly in the experiment during the magnetically quiescent period. The slower response in the simulation may be due to the limitations of the single-temperature collisional heat-flux model or the simplifications of the 
injector waveform. Nonetheless, the 3D simulations reproduce nearly all of the important characteristics of the experiment with physical parameters chosen to model these discharges and without any fitting parameters. The results of this numerical study call attention to the importance of transient effects in SSPX discharges, despite the use of DC current-drive, which can be sustained indefinitely in principle. When the second current pulse is applied, the configuration appears to be sustained because the time required for the amplified poloidal flux to decay is much longer than the injector pulse-length. However, the plasma current is driven inductively by the decay of poloidal flux generated earlier in the discharge by predominantly $n=1$ MHD activity. According to the simulations, the primary role of the second current pulse is to tailor the $q$-profile with respect to avoiding harmful MHD activity that is resonant in the decaying poloidal flux. The correlation of performance-limiting $n>1$ modes with the appearance of corresponding safety-factor values in fitted MHD equilibria has also been noted recently $[16,24]$ for the experiment.

The formation of magnetic flux-surfaces during decay from chaotic and open magnetic configurations was observed in $0-\beta$ computations with a fixed resistivity profile $[9,25]$. However, here it has been demonstrated for the first time with consistent energy transport modeling and nonlinear interactions between the temperature and magnetic field through temperature-dependent resistivity. The increasing temperatures during free decay show that the spheromak system tends to self-organize a coherent and energy-confining structure when it is not sustained, even without external control. The extent to which external controls, such as the second injector-current pulse, can benefit cyclical operation remains to be determined. Already, temperatures exceeding $200 \mathrm{eV}$ have been obtained in SSPX by extending the second current 
pulse and carefully adjusting its amplitude [16]. Simulations of the newer current-drive strategies are underway.

The authors wish to thank Simon Woodruff for many valuable discussions regarding SSPX operations and physics and Ken Fowler for pointing-out the relevance of collisional closures when modeling DC injected spheromaks. Some of the reported simulations have been performed at the National Energy Research Scientific Computing Center. The spheromak research is supported by the U.S. Department of Energy through grant DE-FG02-02ER54687 at the University of Wisconsin-Madison and contract W7405-ENG-48 at Lawrence Livermore National Laboratory.

[1] L. Lindberg and C. T. Jacobsen, Phys. Fluids 7, S44 (1964).

[2] W. C. Turner et al., Phys. Fluids 26, 1965 (1983).

[3] T. R. Jarboe et al., Phys. Rev. Lett. 51, 39 (1983).

[4] T. G. Cowling, Mont. Not. R. Astron. Soc. 94, 39 (1934).

[5] A. H. Boozer, Phys. Fluids B 5, 2271 (1993).

[6] J. B. Taylor, Phys. Rev. Lett. 33, 1139 (1974).

[7] M. G. Rusbridge, Plasma Phys. Control. Fusion 33, 1381 (1991).

[8] A. al-Karkhy et al., Phys. Rev. Lett. 70, 1814 (1993).

[9] C. R. Sovinec, J. M. Finn, and D. del-Castillo-Negrete, Phys. Plasmas 8, 475 (2001).

[10] J. M. Finn, C. R. Sovinec, and D. del-Castillo-Negrete, Phys. Rev. Lett. 85, 4538 (2000).

[11] E. B. Hooper, R. H. Cohen, and D. D. Ryutov, J. Nucl. Mat. 278, 104 (2000).

[12] R. W. Moses, R. A. Gerwin, and K. F. Schoenberg, Phys. Plasmas 8, 4839 (2001).

[13] T. R. Jarboe et al., Phys. Fluids B 2, 1342 (1990). 
[14] E. B. Hooper, L. D. Pearlstein, and R. H. Bulmer, Nucl. Fusion 39, 863 (1999).

[15] H. S. McLean et al., Phys. Rev. Lett. 88, 125004 (2002).

[16] H. S. McLean et al., in Proceedings of the $30^{\text {th }}$ EPS Conference on Contr. Fusion and Plasma Phys., St. Petersburg, Russia, ECA Vol. 27A, 3.230 (2003).

[17] S. I. Braginskii, Reviews of Plasma Physics, edited by M. A. Leontovich, Vol. 1, p. 205 (Consultants Bureau, New York, 1965).

[18] G. W. Hammett and F. W. Perkins, Phys. Rev. Lett. 64, 3019 (1990).

[19] E. D. Held et al., Phys. Plasmas 8, 1171 (2001).

[20] C. R. Sovinec et al., J. Comput. Phys. 195, 355 (2004).

[21] S. C. Hsu and P. M Bellan, Phys. Rev. Lett. 90, 215002 (2003).

[22] B. W. Stallard et al., Phys. Plasmas 10, 2912 (2003).

[23] C. R. Sovinec and S. C. Prager, Phys. Plasmas 3, 1038 (1996).

[24] S. Woodruff, private communication.

[25] R. H. Cohen et al., Nucl. Fusion 43, 1220 (2003). 
(a)

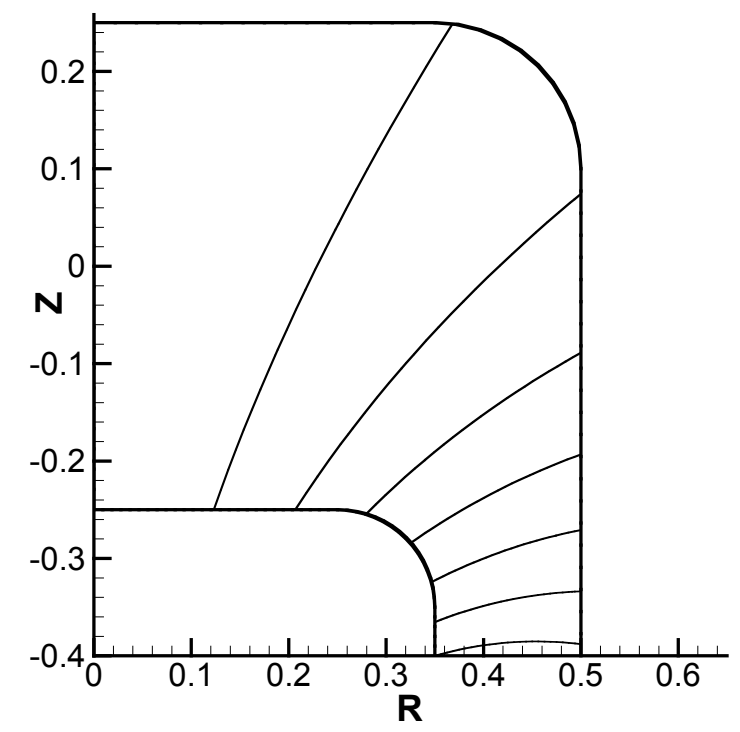

(c)

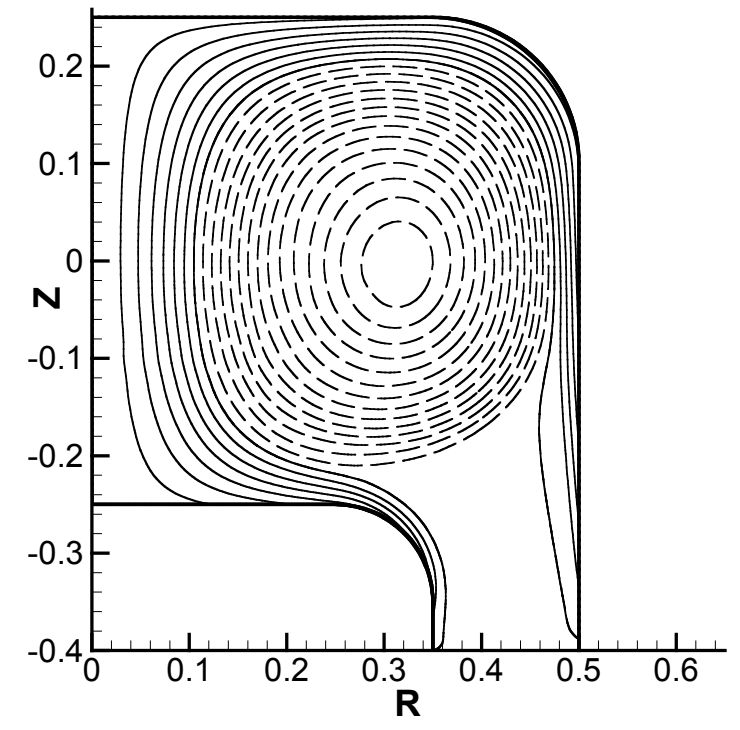

(b)

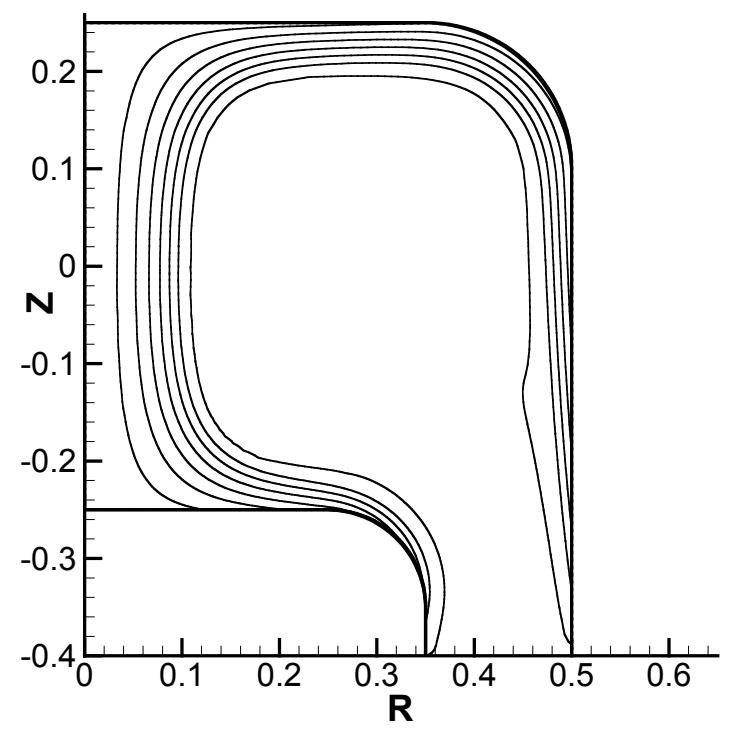

(d)

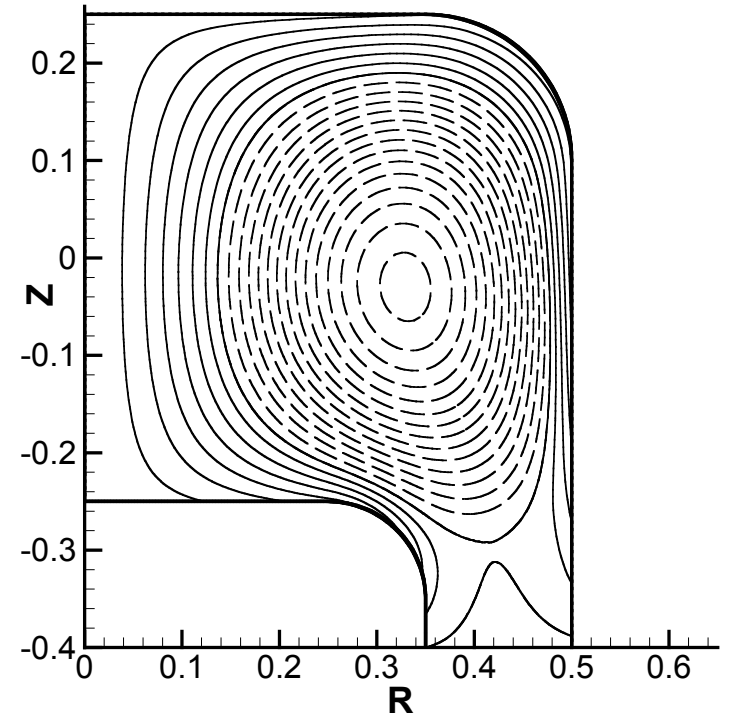

FIG. 1. Contour plots of the poloidal magnetic flux function for the toroidally symmetric component of magnetic field for (a) the initial vacuum distribution, (b) the pinched state at $t=0.08 \mathrm{~ms}$ before becoming unstable, (c) the relaxed state with $I_{i n}=400 \mathrm{kA}$ at $t=0.12 \mathrm{~ms}$, and (d) the partially driven state at $t=1.2 \mathrm{~ms}$. Dashed contour levels indicate poloidal flux converted from toroidal flux by MHD activity. 


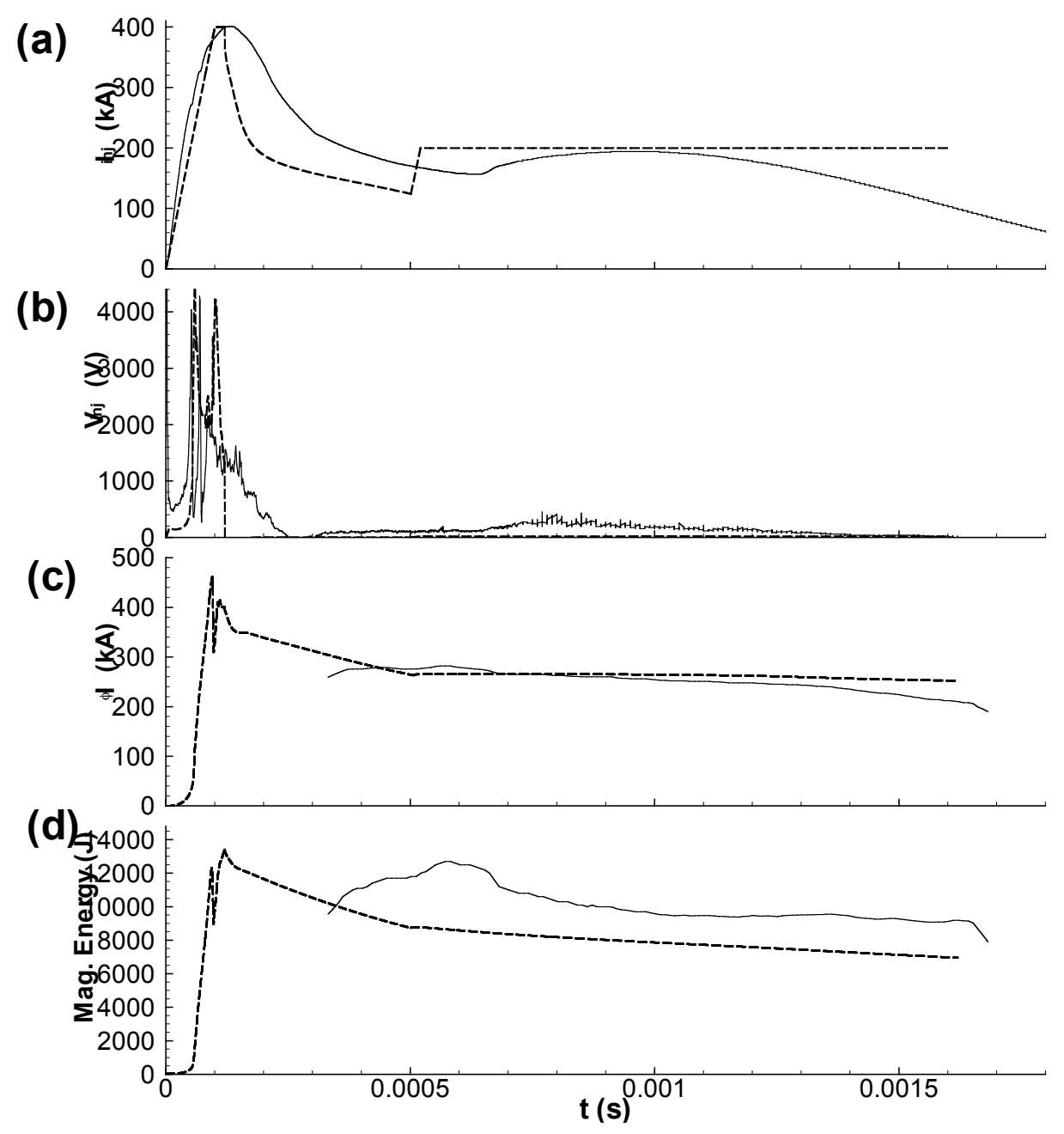

FIG. 2. Evolution of (a) injector current, (b) injector voltage, (c) toroidal current, and (d) stored magnetic energy from SSPX discharge 4624 (solid traces) and the simulation (dashed traces). The SSPX results in (c) and (d) are based on a sequence of fitted MHD equilibria. 
(a)

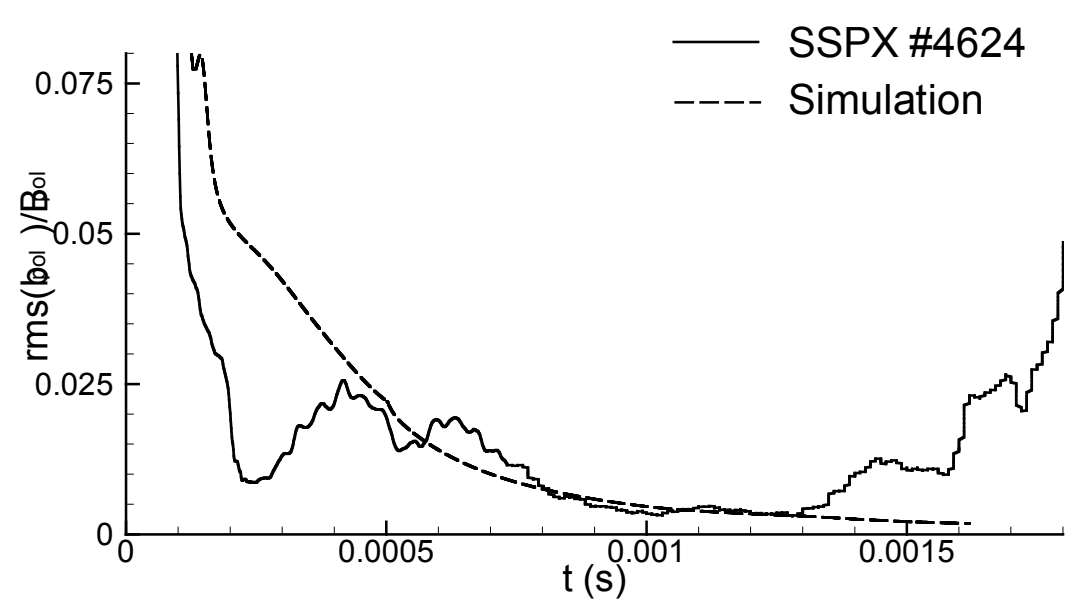

(b)

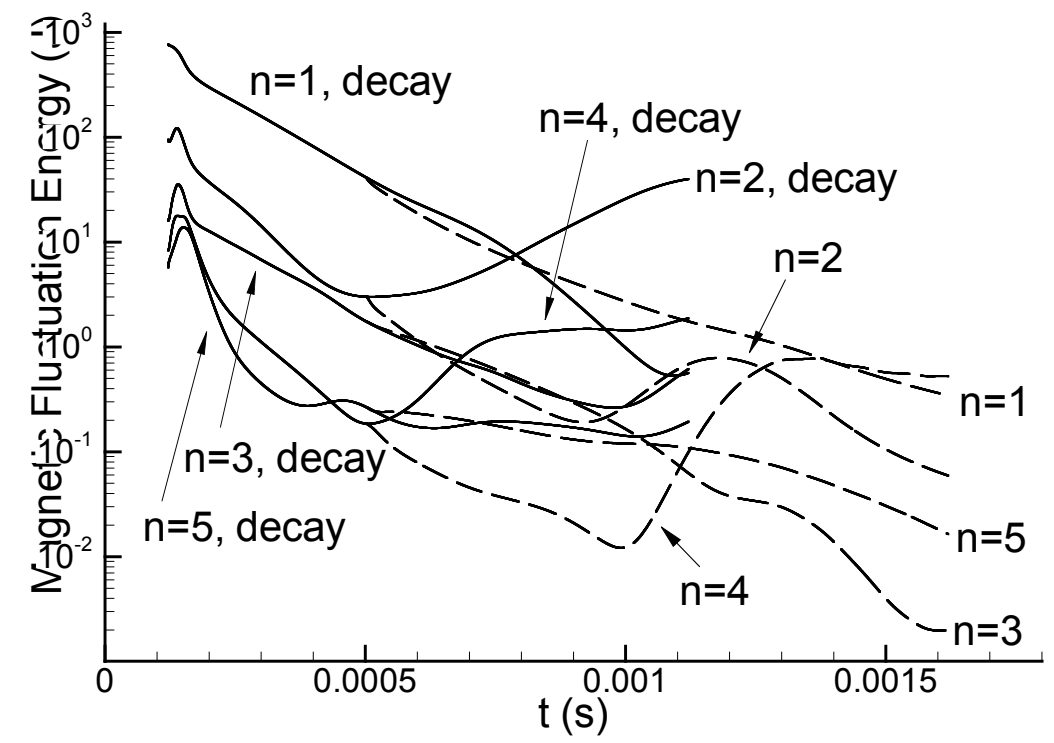

FIG. 3. Magnetic fluctuation information of (a) rms of the poloidal component of magnetic fluctuations relative to the equilibrium poloidal component at the outboard midplane probe location $(R=0.5, Z=0$ in Fig. 1$)$ from the experiment and simulations, and (b) volume-integrated magnetic fluctuation energy from the simulations by Fourier component. The energy plot shows the fluctuations with (dashed lines) and without (solid lines) the second current pulse. 

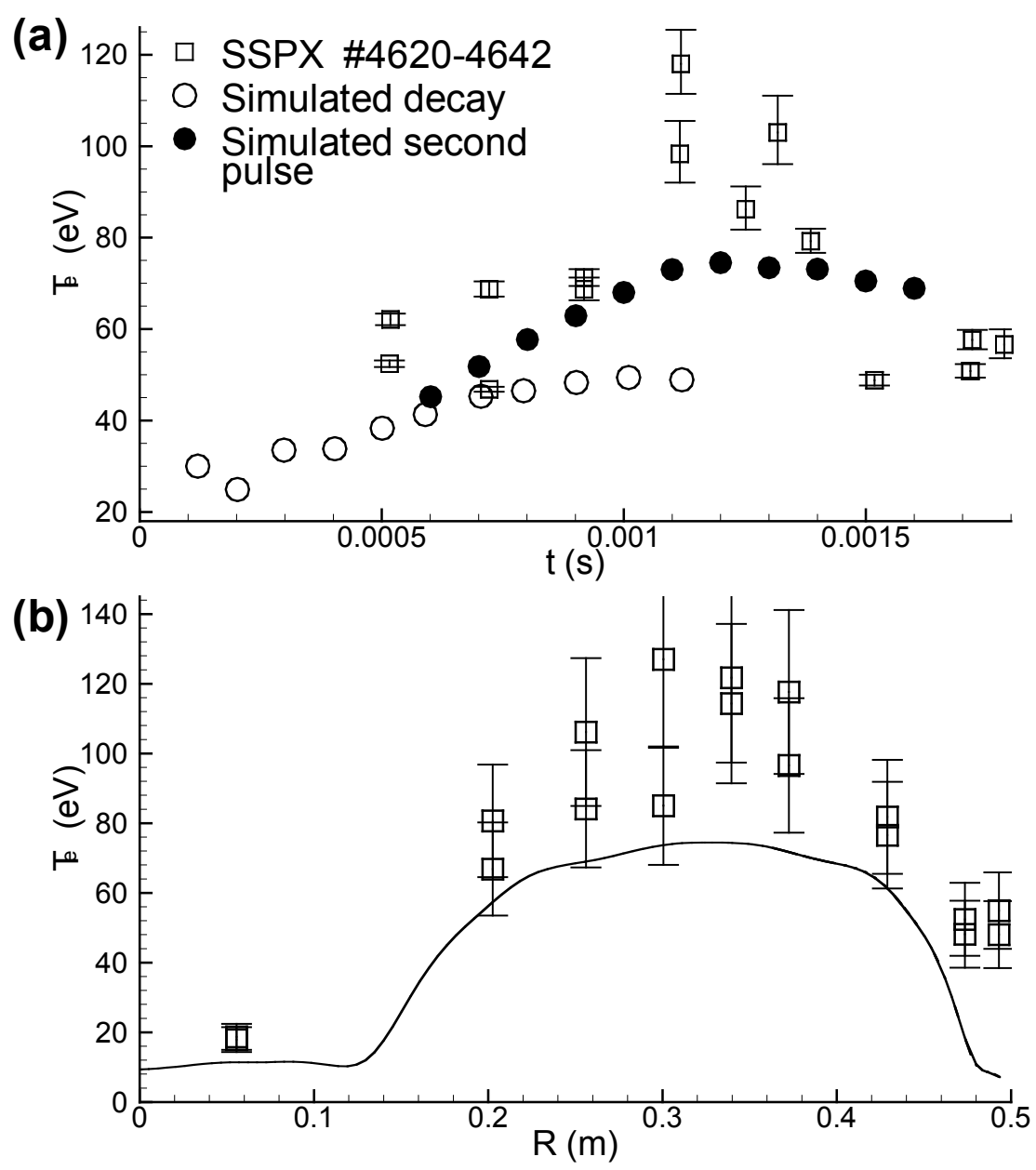

FIG. 4. Comparisons of electron temperature measured with Thomson scattering in SSPX discharges 4620-4642 with simulation results on $\langle T\rangle$. The two plots show (a) temperature evolution central to the amplified poloidal flux and (b) midplane temperature profiles from $t=1.1$ $\mathrm{ms}$ and $t=1.2 \mathrm{~ms}$ in the experiment and simulation, respectively. The simulations with and without the second current pulse are identical for $t \leq 0.5 \mathrm{~ms}$. 


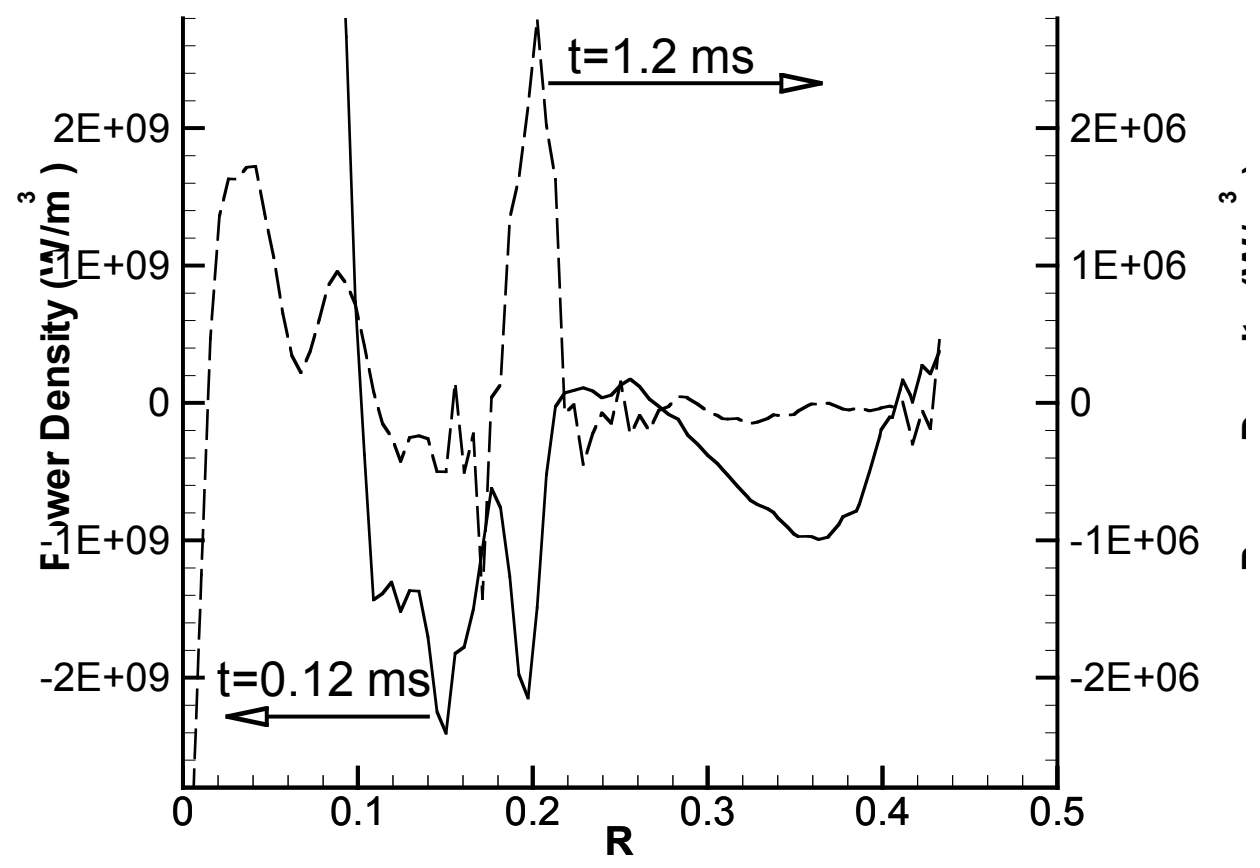

FIG. 5. Magnetohydrodynamic dynamo power density, $-\langle\mathbf{v} \times \mathbf{b}\rangle \cdot\langle\mathbf{J}\rangle$, along the midplane $(Z=0)$ at the end of the first current pulse $(t=0.12 \mathrm{~ms})$ and during the second current pulse $(t=1.2 \mathrm{~ms})$. 
(a)

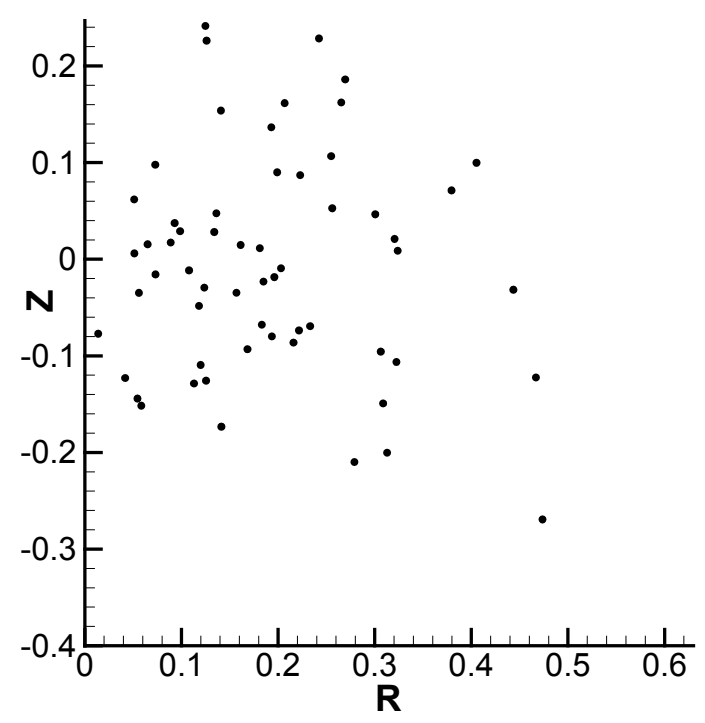

(c)

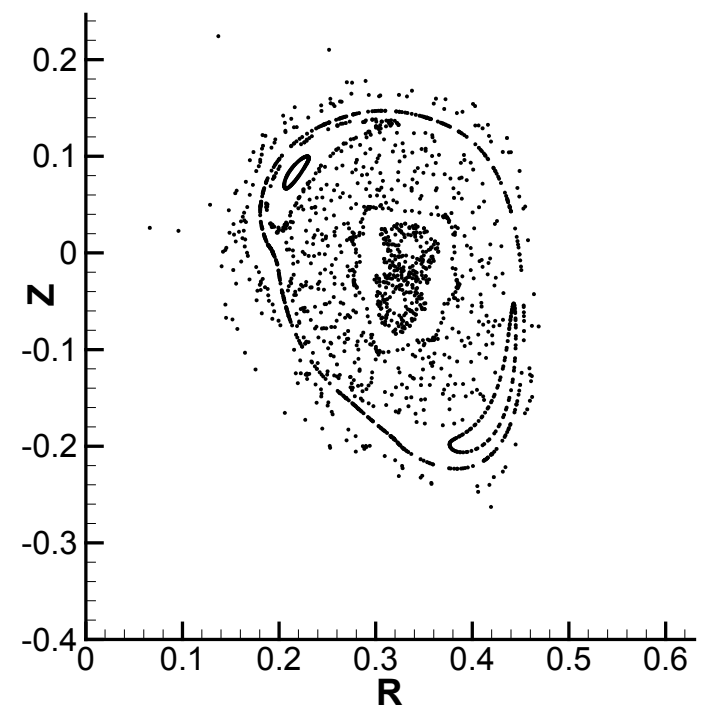

(b)

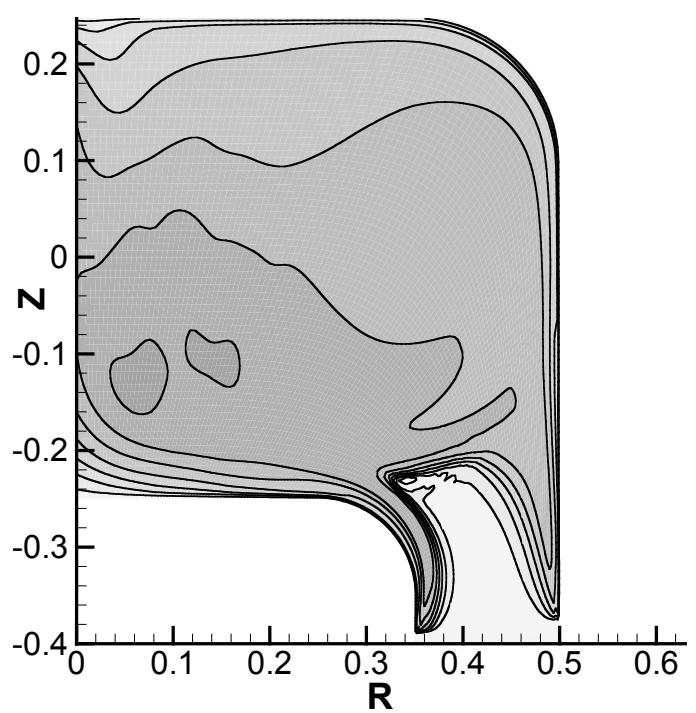

(d)

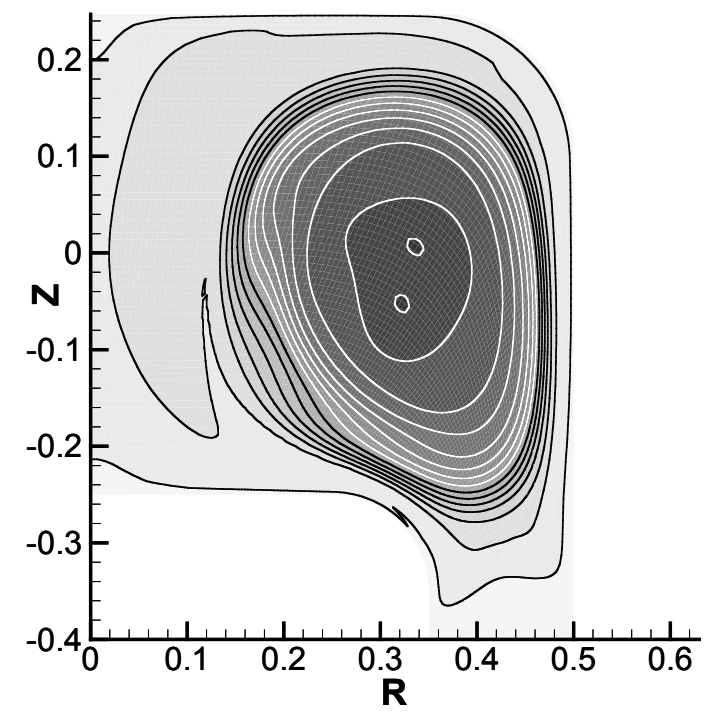

FIG. 6. Magnetic puncture plots and contours of constant temperature at the end of the first current pulse and during the second current pulse. The frames show (a) magnetic punctures at $t=0.12 \mathrm{~ms}$, (b) temperature at $t=0.12 \mathrm{~ms}$, (c) magnetic punctures at $t=1.2 \mathrm{~ms}$, and (d) temperature at $t=1.2 \mathrm{~ms}$. Shading in the two temperature plots is for the same range of 0 to $75 \mathrm{eV}$, and contour levels for $T \geq 40 \mathrm{eV}$ are indicated by white lines. 


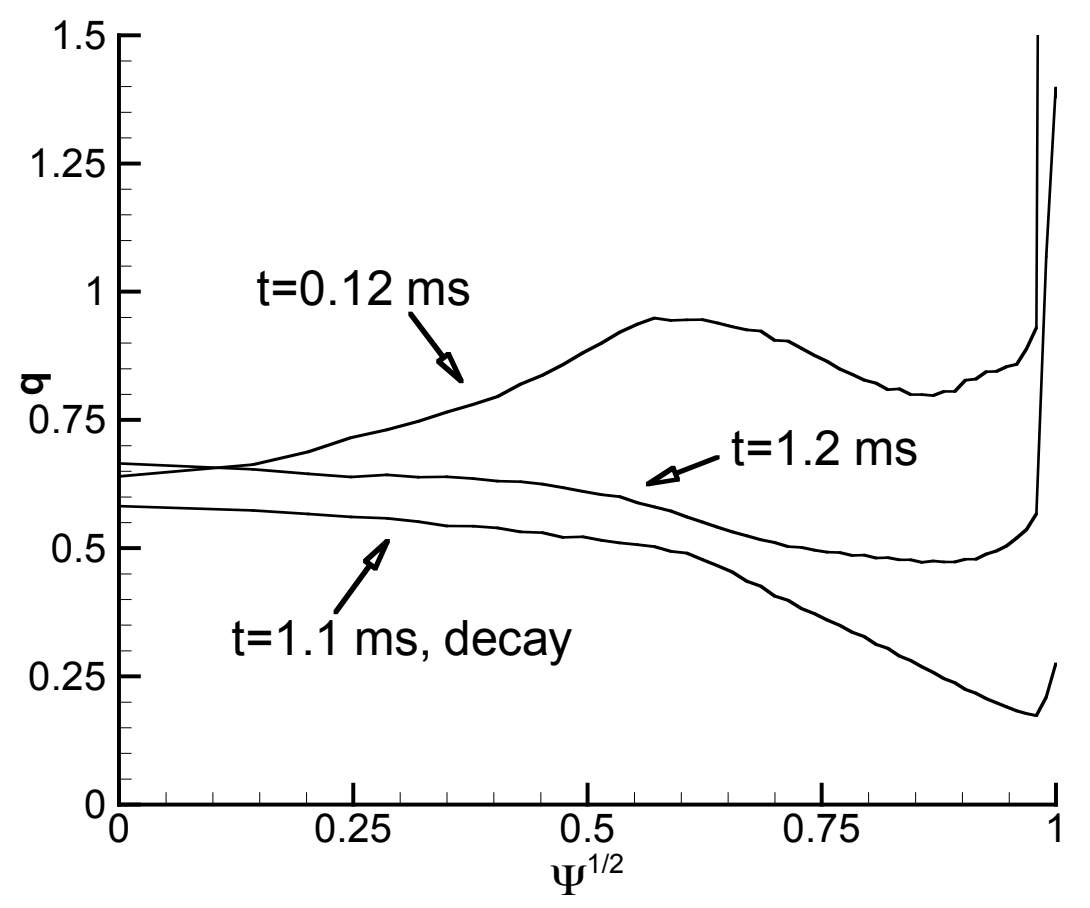

FIG. 7. Safety factor profiles as a function of the square root of normalized poloidal flux. The computation is based on the toroidally symmetric component of magnetic field over the range of flux generated by the MHD activity (the dashed-contour region in Figs. 1c-d). The trace labeled " $t=1.1 \mathrm{~ms}$, decay" is taken from the extended-decay computation, which does not have a second current pulse. 